\title{
A Pricing Mechanism for Scalable Video Delivery ${ }^{1}$
}

\author{
A. Krishnamurthy, T.D.C. Little, and D. Castañon \\ Department of Electrical and Computer Engineering \\ Boston University, Boston, Massachusetts 02215, USA \\ (617) 353-9877, (617) 353-6440 fax \\ tdcl@bu.edu
}

MCL Technical Report 10-01-1995

\begin{abstract}
Many video applications exhibit tolerance to continuous media scaling. Scaling is acceptable due to human tolerance to degradation in picture quality, frame loss and end-toend delay. CM scaling enables the network to utilize its resources efficiently for supporting additional customers and to increase its revenue. However, due to quality degradation, users will not be willing to tolerate scaling unless it is coupled with monetary or availability incentives.

In this paper we propose a pricing policy and a corresponding admission control scheme for scalable video applications. The pricing policy is two-tiered, based on a connection setup component and a scalable component. Connections which are more scalable are charged less but are more liable to be degraded. The proposed policy trades off performance degradation with monetary incentives to improve user benefit and network revenue, and to decrease the blocking probability of connection requests. We demonstrate by means of simulation that this policy encourages users to specify the scalability of an application to the network.
\end{abstract}

Keywords: Scalable video delivery, pricing policy, real-time networks, connection-oriented services, protocols, video-on-demand.

\footnotetext{
${ }^{1}$ In Multimedia Systems, 1996. This work is supported in part by Motorola Codex through the UPR Program and the National Science Foundation under Grant No. IRI-9211165. Portions of this work were presented at the 2nd IEEE Intl. Workshop on Community Networking.
} 


\section{Introduction}

The evolution of computing and networking technology in recent years has enabled the development and support of exciting new distributed multimedia applications (e.g., videoon-demand, distance learning, and video conferencing) which are anticipated to be available to end users on a large scale. Networks supporting Video-on-Demand (VOD) applications will allow users to retrieve and display huge amounts of video data from distributed file servers and sources in a real-time fashion. There are two approaches to the transfer and play-out of such data: transferring all the data ahead of time and then playing them out from local memory (e.g., as done by the HyperText Transport Protocol), or transferring data continuously while playing them out. The latter approach has many advantages given the large amount of data most video applications generate, but is also more difficult to implement due to the real-time nature of VOD applications.

A common approach to guaranteeing adequate quality of presentation during delivery is to reserve sufficient network resources for each individual connection [3]. The problem of efficient allocation of valuable network resources is made significant by the large volume of data coupled with the bursty nature of compressed video. When resources are scarce, the data rate of the connection can be adapted by scaling the data stream [2]. Many video applications are scalable because of human tolerance to degradation in picture quality, frame loss and end-to-end latency, provided the quality of playout is above some perceptual threshold. Tolerance to degradation depends on both the application and the user. For example, in a distance learning application, where audio is more important than video, the user may tolerate the occasional loss of a frame. On the other hand, an application playing out a movie may not tolerate any losses. Video scalability can be translated to a reduction in resource requirements for the corresponding connections. For example, tolerance to large end-to-end latency allows data to be smoothed by buffering before transmission in the network. Similarly, tolerance to picture quality degradation allows encoding parameters to be modified to yield lower data rates. We scale by degradation of image quality, dropping frames, and using smoothing buffers at the source to reduce the resource requirements for connections. Thus, the application can specify a range of resource requirements (ideal and minimum acceptable) to the network during connection establishment. The ideal requirement is needed for reliable delivery of the original data stream while the minimum acceptable requirement is needed for delivering the scaled data stream.

Table 1 shows the ideal and minimum acceptable bandwidth requirements for four 10-minute M-JPEG encoded video sequences with scaling parameters chosen randomly. ${ }^{2} Q$

\footnotetext{
${ }^{2} \mathrm{~A}$ uniform distribution was used and skewed towards the more probable scaling parameters, e.g., a
} 
Table 1: Bandwidth Requirements for Experimental M-JPEG Video Sequences

\begin{tabular}{|r||r||r||r||r||r||r|}
\hline Clip & $\mathbf{Q}$ & $\mathbf{d}$ \% & $d_{c}$ & $\mathbf{D}(\mathbf{m s})$ & $b_{h}(\mathbf{M b} / \mathbf{s})$ & $b_{l}(\mathbf{M b} / \mathbf{s})$ \\
\hline 3 & 75 & 7 & 2 & 40 & 3.94 & 1.16 \\
\hline 4 & 200 & 12 & 4 & 140 & 3.40 & 0.45 \\
\hline 1 & 50 & 2 & 1 & 10 & 3.32 & 1.59 \\
\hline 3 & 75 & 6 & 2 & 30 & 3.94 & 1.20 \\
\hline 2 & 150 & 11 & 3 & 100 & 2.24 & 0.55 \\
\hline 1 & 30 & 1 & 1 & 1000 & 3.32 & 0.45 \\
\hline 1 & 125 & 11 & 3 & 70 & 3.32 & 1.55 \\
\hline 2 & 250 & 14 & 4 & 140 & 2.24 & 0.42 \\
\hline 4 & 50 & 4 & 1 & 10 & 3.40 & 1.48 \\
\hline 3 & 100 & 8 & 2 & 60 & 3.94 & 0.93 \\
\hline 4 & 150 & 11 & 3 & 100 & 3.40 & 0.58 \\
\hline 3 & 30 & 1 & 1 & 20 & 3.94 & 2.06 \\
\hline
\end{tabular}

is the quality compression factor, ${ }^{3} d$ is the percentage of dropped frames, $d_{c}$ is the number of consecutive frame drops allowed, $D$ is the latency $(m s)$, and $b_{h}$ and $b_{l}$ are the ideal and minimum acceptable bandwidth requirements $(\mathrm{Mb} / \mathrm{s})$. Here, we have assumed that scaling is performed at the source and that bandwidth is the only network resource under consideration; such an assumption is justified for single hop networks with sufficient buffering at the source and destination, and for multihop networks under certain conditions [6]. We term this range of resource requirements the "admissible region;" if resource availability in the network is greater than the minimum acceptable requirement, the connection can be admitted [5].

The "admissible region" can be translated to network gains by means of a dynamic connection establishment mechanism [5, 4] that allows renegotiation. If sufficient resources are not available to admit a connection, it can be scaled down within the specified range to enable connectivity while providing a quality above the specified threshold. Furthermore, existing connections may be scaled down to free up resources to admit new connection requests. Clearly, the employment of such a mechanism increases network connectivity, utilization and revenue. However, users suffer quality degradation when applications are scaled down. In the absence of any incentive to specify scalability, users will always request the best possible quality, specifying a large resource demand to the network. Furthermore, even if scalability is specified, the network has no incentive to reserve resources to support connections beyond the minimum specified requirement. Such user and network behavior can lead to inefficient allocation of valuable network resources.

quality factor in the range 30-125 was chosen with twice the probability as that in the range 125-250.

${ }^{3}$ The quality factor is a measure of quantization used in the JPEG encoding scheme. A larger value of $Q$ indicates higher compression and poorer quality. 
We propose a pricing policy for network resources to overcome these problems. The proposed policy provides monetary incentives to offset performance degradation to the user and makes the revenue earned by the network commensurate with the quality delivered. We show by means of simulation that the policy encourages users to specify application scalability to the network. Moreover the network is provided with monetary incentives to support connections at a quality better than the minimum specified when resources are available.

While recent research efforts have focused on solving a number of technological issues in the networking and operating system arenas, little work has been reported in the literature on the development of an appropriate pricing structure for scalable VOD services. Research on pricing issues has focussed on both connectionless transfers on the Internet $[1,7,11,10]$, and reservation-based connection-oriented transfers [8, 9]. MacKie-Mason and Varian [7] propose a pricing policy which charges more during periods of congestion (when bandwidth is a scarce resource) and very little during periods of light load. Cocchi et al. [1] propose a scheme to maximize user satisfaction in a connectionless environment. In a reservation based connection oriented scheme, prices should be based on the resources reserved, and not on the actual volume of traffic transferred [8].

In current literature, scalability and pricing have been studied independently for the provision of integrated services. We contend that these issues complement each other for networks supporting VOD applications. Our work focuses on the relationship between performance and monetary issues from both the user's and network provider's perspectives. The formulation of a pricing policy encompasses a variety of social, regulatory, economic and performance issues. However, in our formulation, we concentrate on utilization and performance issues within the network and ignore other factors. We discuss the proposed pricing policy in Section 2. Section 3 describes our simulation models and environment. We present our results in Section 4. Section 5 concludes the paper.

\section{Proposed Pricing Policy}

The employment of an appropriate pricing policy is essential if benefits from scaling gains are to be utilized. If all customers are charged a fixed amount, customers will always demand the highest possible quality of service. There is no incentive for them to specify application scalability. On the other hand, the network will always serve each connection at its lowest bandwidth even if excess bandwidth is available to provide a better quality. A suitable pricing structure should provide an incentive for the network to scale up connections and utilize excess bandwidth, while encouraging users to request only the resources they need. 


\subsection{Pricing Policy}

The pricing scheme should encourage users to specify the maximum possible scalability to the network when they maximize their individual benefit. The network can then use this scalability to maximize its revenue. Another objective in developing the pricing policy is to decrease the blocking probability of connection requests. A suitable pricing structure should provide an incentive for the network to scale up connections and utilize excess bandwidth. Furthermore, the revenue collected should be proportional to the amount of bandwidth reserved.

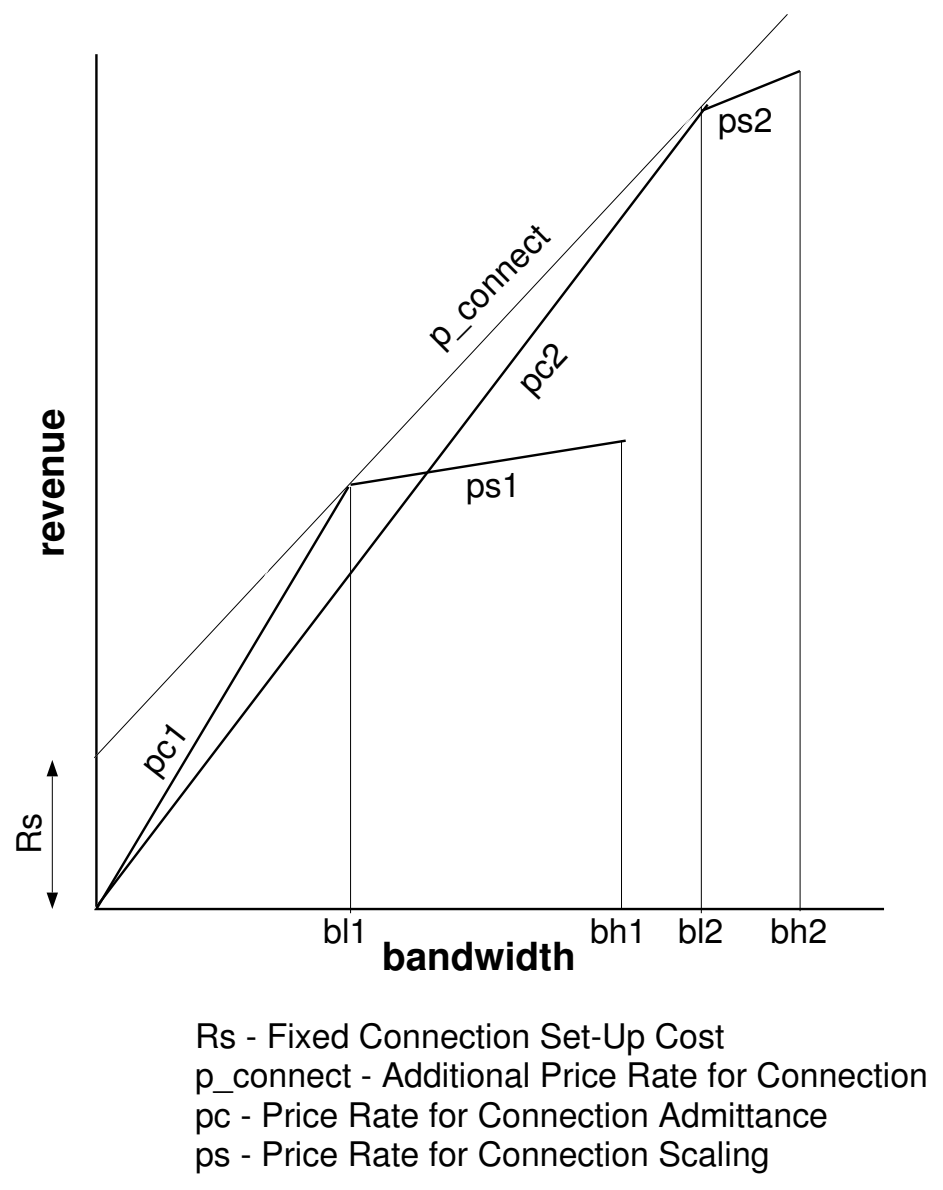

Figure 1: Proposed Pricing Model

Considering these objectives, we propose a pricing structure illustrated in Fig. 1 which plots the revenue obtained against bandwidth allocations for two connections with different requirements. The pricing structure has two tiers corresponding to connection setup and scaling. ${ }^{4}$ All prices rates are per unit bandwidth per unit time. The connection

\footnotetext{
${ }^{4}$ Note that the price rates correspond to the slopes of the curves in the figure.
} 
set-up cost consists of two fixed components: a connection set-up charge $\left(R_{s}\right)$, and a perunit-bandwidth price rate $\left(p_{\text {connect }}\right)$. $p_{\text {connect }}$ is the price rate for admitting the connection with the minimum acceptable bandwidth. Though the connection set-up prices are the same for all connections, each request sees a different effective price rate for connectivity, depending on the minimum requested bandwidth (indicated by $p_{c i}$ in the figure). The price rate component of the scalable region $\left(p_{s}\right)$ is inversely proportional to the scalability of the connection (i.e., directly proportional to $\frac{b_{l}}{b_{h}}$ ), and is always lower than $p_{\text {connect }} \cdot p_{s}$ is the price rate charged for scaling the connection up beyond $b_{l}$. In the proposed policy the cost consists of three components corresponding to the connection set up cost $\left(R_{s}\right)$, price for connectivity $\left(p_{\text {connect }}\right)$, and price for the scalable region $\left(p_{s}\right)$. For a bandwidth allocation $x$, the cost of the connection is:

$$
C(x)= \begin{cases}R_{s}+c \times\left(p_{\text {connect }} \times b_{l}+p_{s} \times\left(x-b_{l}\right)\right), & b_{l} \leq x \\ 0, & b_{l}>x\end{cases}
$$

where $c$ is a weighting factor introduced to keep the cost values within a reasonable range for simulation purposes.

\subsection{Admission Control}

We assume that the network will employ an admission control algorithm to maximize its revenue and therefore its profit, assuming fixed capacity and cost to provide this capacity. In the proposed policy, each connection has two slopes associated with it, one for connectivity $\left(p_{c}\right)$ and one for scalability $\left(p_{s}\right)$. We call these slopes the "connectivity" and "scalability" slopes. Network revenue is maximized if bandwidth is allocated to connections in the higher slope regions. We consider two scenarios of admission control. In the static scenario, the admission control algorithm must choose the connections to admit from a group of requests and compute their bandwidth allocations. ${ }^{5}$ To implement admission control, the network sorts the $p_{c}$ and $p_{s}$ values for the requests in decreasing order and allocates bandwidth starting with the largest value. If the value corresponds to a connectivity price rate, the connection is admitted with bandwidth $b_{l}$. If the value corresponds to a scalability rate, the connection is scaled up $\mathrm{up}^{6}$ to a bandwidth allocation of $b_{h}$ specified in the corresponding request. This is done until there is no bandwidth to allocate or all connections have been scaled up. Note that this policy is heuristic rather than optimal. However, it is simple and leads to the optimal revenue in most cases. $^{7}$

\footnotetext{
${ }^{5}$ Note that the relationship $p_{c}>p_{s}$ always holds.

${ }^{6}$ This is done only if the connection has already been accepted.

${ }^{7}$ Without scalability the admission control algorithm reduces to a greedy heuristic for a bin-packing problem.
} 
In the dynamic scenario, requests for connection establishment and release are received by the admission control algorithm over time. On receiving a request, the algorithm attempts to admit the connection at the minimum bandwidth. If sufficient bandwidth is not available, the algorithm checks to see if the required bandwidth can be freed by scaling down existing connections. If this test fails, the request is rejected. If it passes, existing connections are scaled until sufficient resources are freed. Connections are scaled down in increasing order of scalability slopes. Once the connection is admitted, the algorithm attempts to scale it up at the expense of connections with lower scalability slopes. When a connection is released, existing connections are scaled up in order of decreasing scalability slopes until all available bandwidth is allocated.

\subsection{Implications}

We make the following observations about our proposed pricing structure which relate to the objectives of our pricing model. We illustrate these observations by means of a simple scenario. Consider two connection requests $A$ and $B$ with $b_{h}$ and $b_{l}$ as specified in Table 2.3. The table also shows the corresponding values of the connectivity price, computed by

$$
p_{c}=\frac{R_{s}+c * p_{\text {connect }}}{b_{l}}
$$

and the scalability price obtained by

$$
p_{s}=\frac{b_{l}}{b_{h}}
$$

Note that the revenue for any connection admitted and allocated bandwidth $x$ can be written as:

$$
C(x)=p_{c} \times b_{l}+p_{s} \times\left(x-b_{l}\right)
$$

For this example, we have assumed $R_{s}=10, p_{\text {connect }}=1$, and $c=12$.

Table 2: Example Scenario

\begin{tabular}{|r||r||r||r||r|}
\hline Request & $b_{h}(\mathbf{M b} / \mathbf{s})$ & $b_{l}(\mathbf{M b} / \mathbf{s})$ & $p_{c}$ & $p_{s}$ \\
\hline$A$ & 3.94 & 1.16 & 18.97 & 0.29 \\
\hline$B$ & 2.24 & 0.55 & 40.0 & 0.25 \\
\hline
\end{tabular}

- Connections with lower minimal acceptable bandwidth requirements are given higher priorities for connection admission in the static scenario. The effective connection price $p_{c}$ is higher for connections with lower $b_{l}$ due to the fixed set-up cost $R_{s}$. In 
our example, $B$ has a lower minimal bandwidth requirement, and is given priority for admission because it has a higher value of $p_{c}$ than $A$.

- Once connected, applications specifying higher scalability are charged a lower price but are also more likely to be scaled, since their price rate $p_{s}$ is lower. In our example, $B$ is more likely to be scaled because of a lower $p_{s}$.

- Increasing the scalability of an application decreases its blocking probability. For example, if $A$ were being considered for connection and the available bandwidth after scaling existing connections was $1 \mathrm{Mb} / \mathrm{s}$, the request would be rejected. However, if $A$ were more scalable, reducing the value of $b_{l}$ to $1 \mathrm{Mb} / \mathrm{s}$, the connection would be admitted.

- The network increases its revenue by scaling down applications to accept a new request, both by increasing utilization and by receiving additional revenue for the same bandwidth. Consider a scenario in which the network had a total bandwidth of $3 \mathrm{Mb} / \mathrm{s}$, and supports $B$ at $2.24 \mathrm{Mb} / \mathrm{s}$ when request $A$ arrives. The network earns a revenue of 21.67 per unit time for supporting $B$. To accommodate $A$ the connection scales down $B$ with the admission control algorithm such that it now supports $B$ at the minimum bandwidth of $0.55 \mathrm{Mb} / \mathrm{s}$ and $A$ at $2.45 \mathrm{Mb} / \mathrm{s}$. The revenue earned by the network now is 45.01 per unit time. Thus, the network benefits by scaling down $B$ to admit $A$.

- The network increases its revenue by scaling up applications when it has unused bandwidth. To illustrate this, consider a scenario when $B$ is supported at the minimum bandwidth, and a connection is released. If the available bandwidth is used to scale up $B$ to its value of $b_{h}$, the revenue earned increases by 5.07 per unit time.

- Customers running applications with higher scalability are charged a lower price for the same bandwidth, while those paying a higher price are less likely to be down-scaled.

\section{Simulation Models and Environment}

We now describe our models for user utility and cost, and describe simulation scenarios and performance parameters.

\subsection{User Utility Function}

The user utility function is a measure of user satisfaction as a function of allocated resources. We assume a model of diminishing returns; the marginal utility to the user diminishes as 
a function of allocated bandwidth. User utility is non-zero only when the connection is admitted (i.e., the allocated bandwidth is greater than $b_{l}$ ). Furthermore, the marginal utility is zero when the allocated bandwidth is $b_{h}$, i.e., the utility does not increase with increasing bandwidth allocation at this point. Formally, we define the user utility function for any bandwidth allocation $x$ as:

$$
U(x)= \begin{cases}u \times\left(b_{h} \times x-\frac{x^{2}}{2}\right)+U_{c}, & \text { if } b_{l} \leq x \leq b_{h} \\ 0, & \text { if } x<b_{l} \\ u \times \frac{b_{h}^{2}}{2}+U_{c}, & \text { if } x>b_{h}\end{cases}
$$

Here, $U_{c}$ is an additive constant reflecting the utility for connectivity, and $u$ is a weighting factor introduced to keep utility values within reasonable ranges for our study. An example of a utility function $\left(U_{c}=0, u=1\right)$ with $b_{h}=3.94 \mathrm{Mb} / \mathrm{s}$ and $b_{l}=0.96 \mathrm{Mb} / \mathrm{s}$ is shown in Fig. 2.

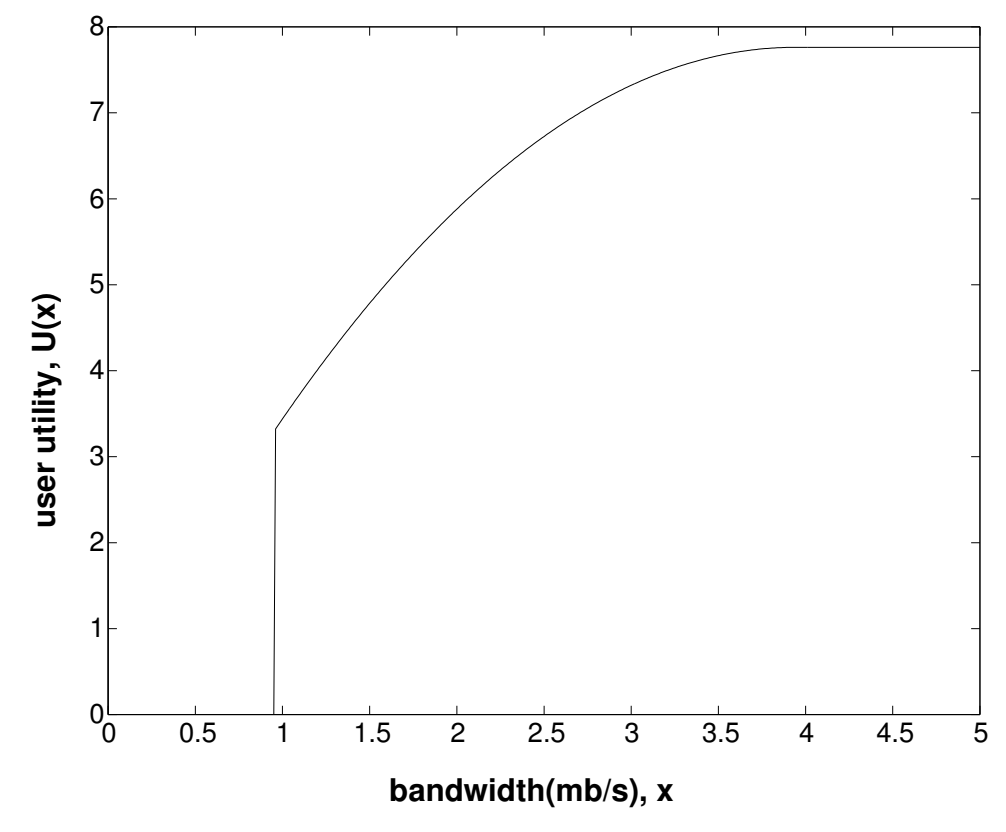

Figure 2: An Example Utility Function

\subsection{Cost Functions}

The user cost per connection is dependent on the pricing policy used by the network. In a fixed cost policy the cost for a connection is fixed and independent of the bandwidth allocated:

$$
C(x)= \begin{cases}\mathrm{C}, & \text { if } b_{l} \leq x \\ 0, & \text { otherwise }\end{cases}
$$


In the proposed policy the cost is:

$$
C(x)= \begin{cases}R_{s}+c \times\left(p_{\text {connect }} \times b_{l}+p_{s} \times\left(x-b_{l}\right)\right), & b_{l} \leq x \\ 0, & b_{l}>x\end{cases}
$$

as described earlier.

\subsection{Performance Metrics}

Our objective is to demonstrate that the proposed policy encourages the user to specify the maximum possible scalability to the network during connection establishment. The user specifies bandwidth requirements so as to maximize benefit, which is defined as the utility derived minus cost paid:

$$
B(x)=U(x)-C(x)
$$

We show that applying scalability by means of a dynamic admission control scheme leads to significant user benefit, network revenue and connectivity gains over a fixed non-scalable scheme (the fixed cost scheme). We consider percentage of blocked requests, aggregate user benefit and network revenue (aggregate user cost) as performance metrics in our analysis.

\subsection{Analysis of User Preferences}

While the network adopts admission control algorithms to maximize its revenue, the user tailors the resource requirement specification to maximize benefit. The user optimizes benefit by demanding bandwidth $x$ such that

$$
\begin{gathered}
B^{\prime}\left(x_{o p t}\right)=0 \\
U^{\prime}\left(x_{o p t}\right)=C^{\prime}\left(x_{o p t}\right)
\end{gathered}
$$

With the fixed cost policy, $C^{\prime}(x)=0$, and user utility is maximized when

$$
U^{\prime}\left(x_{\text {opt }}\right)=0
$$

That is,

$$
x_{\text {opt }}=b_{h}
$$

Thus, the user always demands the maximum bandwidth from the network, and has no incentive to provide a scaling range. In this model, we assume that the user is not influenced by the probability of the request being blocked. When the network is congested, the probability of admission increases with a lower specification of bandwidth. This factor is considered by using a model where the user specifies less than the optimally calculated bandwidth. In the 
dynamic scenario, the user can optimize if information about the available bandwidth $\left(b_{a}\right)$ is known. If the optimal bandwidth $\left(b_{h}\right)$ is greater than the available bandwidth, the user specifies $b_{a}$.

In the proposed policy, the user will specify a range $\left(b_{\min }, b_{h}\right)$ to the network so as to maximize the expected benefit. The range specified, $\left(b_{\min }, b_{h}\right)$, is a subset of the entire range (i.e., $\left.b_{l} \leq b_{\text {min }}\right)$. Note that the higher value in the range is always $b_{h}$, since a lower value will increase the cost without changing the utility, thus decreasing the benefit. Depending on the state of the network, connection set-up requests are either allocated the minimum specified bandwidth, the maximum specified bandwidth, or blocked. ${ }^{8}$ We define

$$
\begin{gathered}
P_{\text {min }}=P\left[b_{\text {alloc }}=b_{\text {min }}\right] \\
P_{b}=P[\text { blocked }]
\end{gathered}
$$

where $b_{\text {alloc }}$ is the allocated bandwidth. Thus,

$$
P\left[b_{\text {alloc }}=b_{h}\right]=1-P_{\min }-P_{b}
$$

The expected user utility is then

$$
E[U]=P_{\min } \times U\left(b_{\min }\right)+\left(1-P_{\min }-P_{b}\right) \times U\left(b_{h}\right)
$$

The expected cost is given by

$$
E[C]=P_{\min } \times C\left(b_{\min }\right)+\left(1-P_{\min }-P_{b}\right) \times C\left(b_{h}\right)
$$

The expected user benefit is

$$
E[B]=E[U]-E[C]
$$

Note that in a real system, the values of $P_{\min }$ and $P_{b}$ are not known. However, this analysis proves useful in special cases when the network is lightly or heavily loaded.

In a lightly loaded system, all requests are allocated the maximum bandwidth requested, $b_{h}$, irrespective of the size of the range. In this scenario, $P_{\min }=P_{b}=0$. Thus,

$$
E\left[B\left(b_{\min }\right)\right]=U\left(b_{h}\right)-C\left(b_{h}\right)
$$

The value of $b_{\min }$ which maximizes expected benefit is calculated by

$$
E\left[B^{\prime}\left(b_{\min }\right)\right]=E\left[U^{\prime}\left(b_{\min }\right)\right]-E\left[C^{\prime}\left(b_{\min }\right)\right]=0
$$

\footnotetext{
${ }^{8}$ With our admission control algorithm one request is always allocated a bandwidth between minimum and maximum; we ignore this event in our analysis.
} 
We obtain

$$
E\left[B^{\prime}\left(b_{\text {min }}\right)\right]=-p_{\text {connect }}-1+2 \times \frac{b_{\text {min }}}{b_{h}}
$$

Note that the value of $b_{\min }$ which makes $E\left[B\left(b_{\min }\right)\right]=0$ minimizes it. Furthermore, $p_{\text {connect }}>$

$\frac{b_{\min }}{b_{h}}$, and $\frac{b_{\min }}{b_{h}} \leq 1$, which implies that the value of $b_{\min }$ which maximizes expected benefit is outside the range $\left(b_{l}, b_{h}\right)$. The most optimal value is obtained when $E\left[B\left(b_{\text {min }}\right)\right]$ is most negative, i.e., when $b_{\min }=b_{l}$. Thus, in a lightly loaded system, user benefit is maximized by specifying the entire range since this minimizes the scalability cost rate, and the network will provide full bandwidth service anyway.

If the system is heavily loaded, the user tries to minimize blocking probability. This is again achieved by specifying the entire range; a lower value of $b_{l}$ decreases blocking probability. For intermediate loads, the optimum user specification is not so obvious. If the blocking probability is ignored in the analysis, i.e., $P_{\min }=1$ and $P_{b}=0$

$$
\begin{gathered}
U\left(b_{\min }\right)=u \times\left(b_{h} \times b_{\text {min }}-\frac{b_{\text {min }}^{2}}{2}\right)+U_{c} \\
C\left(b_{\text {min }}\right)=R_{s}+c \times\left(p_{\text {connect }} \times b_{\text {min }}\right) .
\end{gathered}
$$

We calculate the value of $b_{\min }$ by

$$
\begin{gathered}
U^{\prime}\left(b_{\text {min }}\right)=C^{\prime}\left(b_{\text {min }}\right) \\
b_{\text {min }}=b_{h}-\frac{c}{u} \times p_{\text {connect }}
\end{gathered}
$$

If $b_{\min }<b_{l}$, the user specifies the entire range $\left(b_{l}, b_{h}\right)$, i.e., $b_{\min }=b_{l}$.

\subsection{Admission Control}

The admission control algorithm for the proposed scheme has been described in Section 2.2. In the static case, $p_{c}$ and $p_{s}$ values are sorted in decreasing order and bandwidth allocated starting with the largest value. In the dynamic case, the connection is admitted if sufficient bandwidth is available. If this is not the case, existing connections are down-scaled in increasing order of scalability slopes to free up resources. If resources are still not sufficient, the connection is refused. Once the connection is admitted, the algorithm tries to scale it up by scaling down existing connections with lower scalability slopes. When a connection is released, existing connections are scaled up in order of decreasing scalability slopes.

In the fixed cost case the network obtains the same revenue for each connection independent of the bandwidth allocated to it. The network maximizes its revenue by admitting connections with the smallest bandwidth requirements first $\left(b_{h}\right)$. To execute this admission policy in the static case, the network sorts the bandwidth specifications of connections in 
increasing order and admits connections starting with the lowest minimum acceptable bandwidths. In the dynamic case, the connection simply admits connections if it has sufficient bandwidth when the request is received.

\subsection{Simulation Scenarios}

Simulations were performed for two scenarios. In the static scenario, all requests were assumed to arrive at the same instant. The number of requests was varied over different runs. In the dynamic scenario, requests arrived with a Poisson distribution, the rate of which was varied over different runs. The holding time of connections was fixed at 10 minutes. Performance parameters for the dynamic case were measured per unit time. The $b_{l}$ and $b_{h}$ parameters for the requests were chosen randomly from a database based on real video sequences (created in advance) similar to the one in Table 1. Simulations were performed for the fixed cost policy and our proposed scheme. The parameters for the cost and utility functions were chosen such that the user always benefits (i.e., the benefit is always positive) if the connection is admitted. We set $U_{c}=500$ and $u=12$ per unit time (min). In the fixed cost policy, $C(x)=500$. For the proposed policy, $R_{s}=200, c=12$ per unit time (min),

$p_{\text {connect }}=1$, and $p_{s}=\frac{b_{l}}{b_{h}}$. The total available bandwidth was set at $100 \mathrm{Mb} / \mathrm{s}$. The results were obtained by averaging over 100 runs. This scenario models a modest VOD system with an arbitrarily large number of users. Details of database generation can be found in [4].

\section{Results and Discussion}

We first compare the proposed policy with the fixed cost policy in the static case. In this experiment, users ask for the maximum possible bandwidth $\left(b_{h}\right)$ in the fixed cost policy and specify the entire range $\left(b_{l}, b_{h}\right)$ in the proposed policy. Fig. 3 shows the percentage of requests blocked as the number of requests increases.

We see that the fixed cost policy starts blocking much earlier than our policy. Both policies admit connections at $b_{h}$ until the bandwidth is saturated. The fixed cost policy cannot admit connections beyond this point and blocks additional calls, while the proposed policy scales down existing connections to admit more connections. Moreover, we observe that the slope of the curve is greater for the fixed cost policy. The proposed policy can still admit connections after it starts blocking because it admits connections at $b_{l}$ which can be significantly lower than $b_{h}$. This is reflected in Fig. 4 which shows network revenue plotted against number of requests. Note that we are more concerned with the shapes of the curves than their locations. Either curve can be raised by increasing the cost parameters. The fixed cost curve flattens out when blocking starts, indicating that the number of connections 


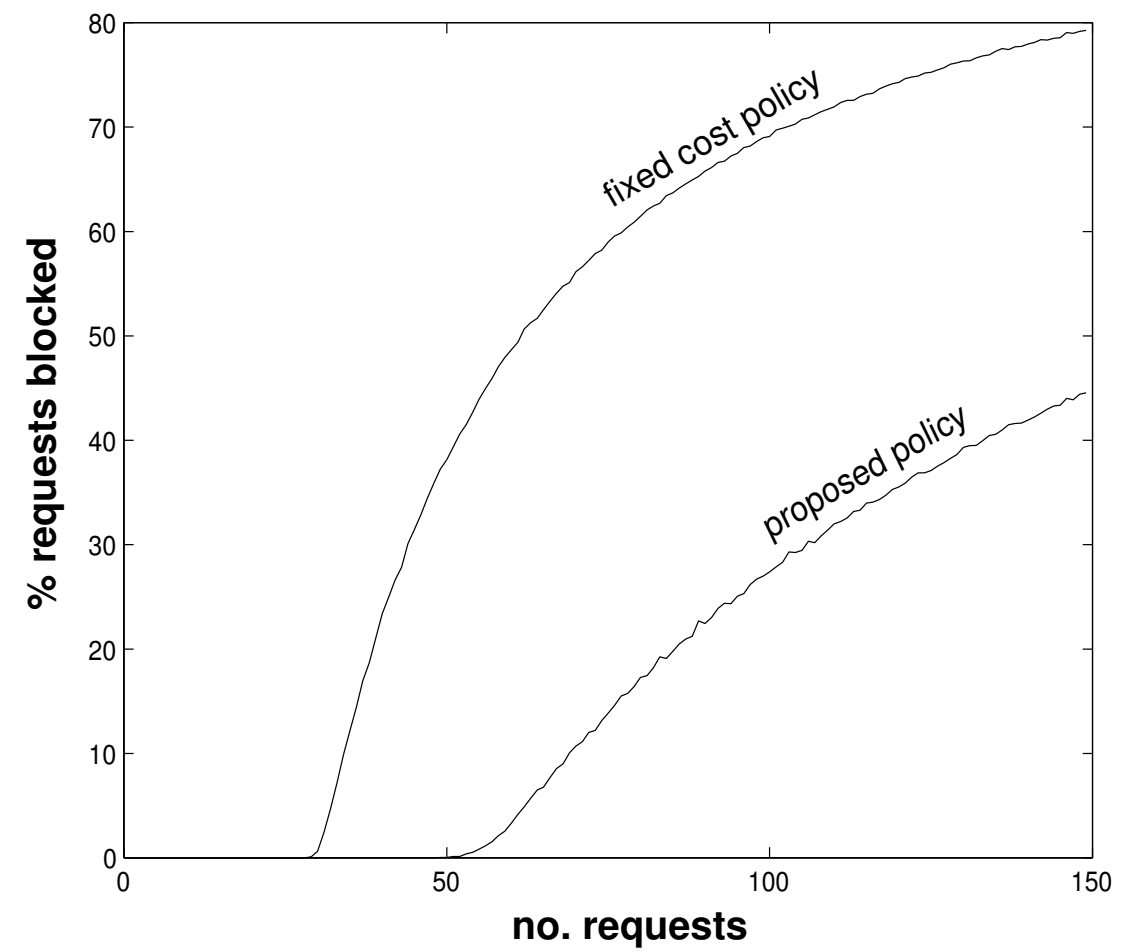

Figure 3: Percentage of Requests Blocked: Static Case 


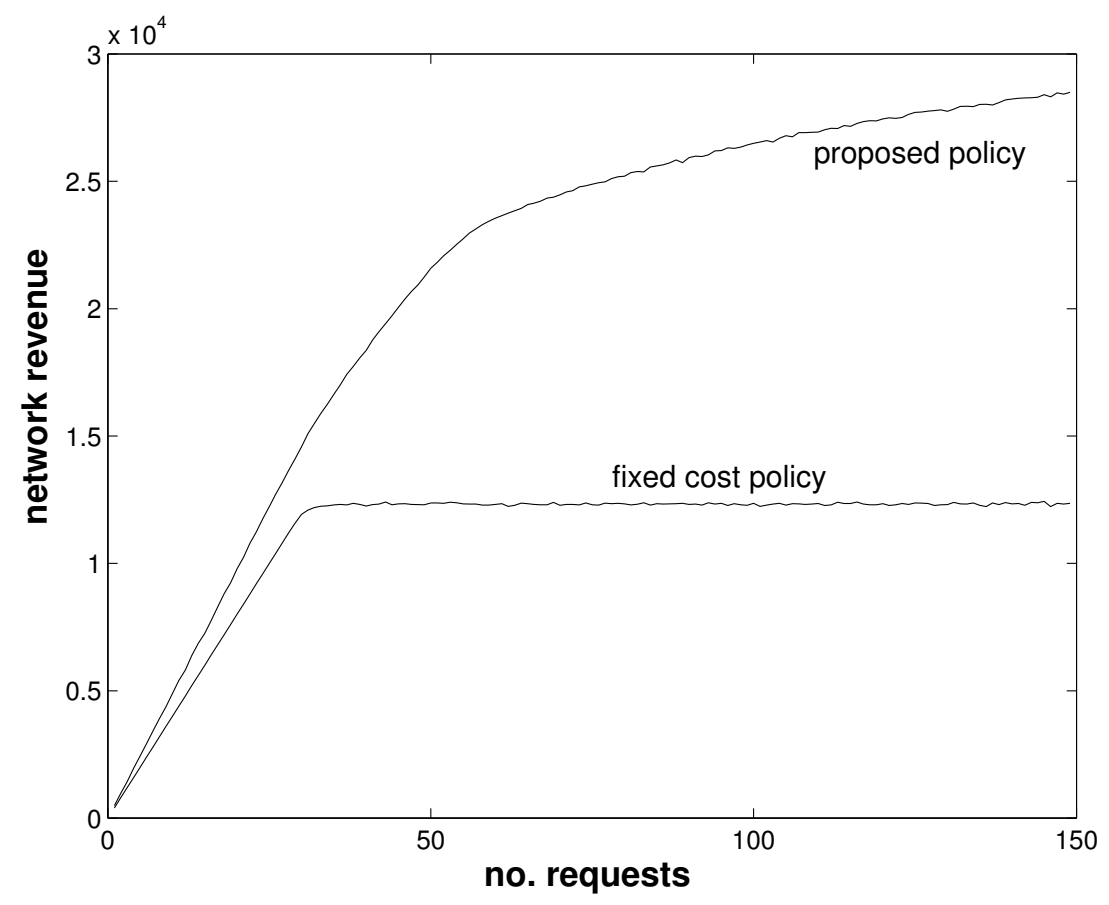

Figure 4: Network Revenue: Static case

increases only slightly once blocking starts. ${ }^{9}$ With the proposed policy, the curve flattens at a higher number of requests (blocking starts later), and increases in the blocking region, indicating that a significant number of requests are still admitted. The loss of revenue due to scaling down connections is more than offset by gains due to admitting more connections. Finally, the user benefit curves are illustrated in Fig. 5. Again, the user benefit curve flattens out for the fixed cost policy. Note that the benefit may actually decrease in the heavily loaded region because connections with lower bandwidth requirements (and therefore utility) are admitted at the same cost. In our example, the curve remains flat because the lower benefit per connection is offset by an increase in the number of connections. In the proposed policy, the decrease in utility per connection due to scaling is offset by a decrease in cost.

If the user lowers the requirement to increase the probability of admission in the blocking region, more requests may be admitted in the fixed price case. However, the benefit per admitted user decreases because the decrease in utility is not offset due to the fixed cost structure. Depending on the actual cost, users may find it more beneficial not to request the connection, leading to a drop in network revenue. From these results, we conclude that the

\footnotetext{
${ }^{9}$ Connections with lower resource requirements are admitted first, so that more connections can be admitted.
} 


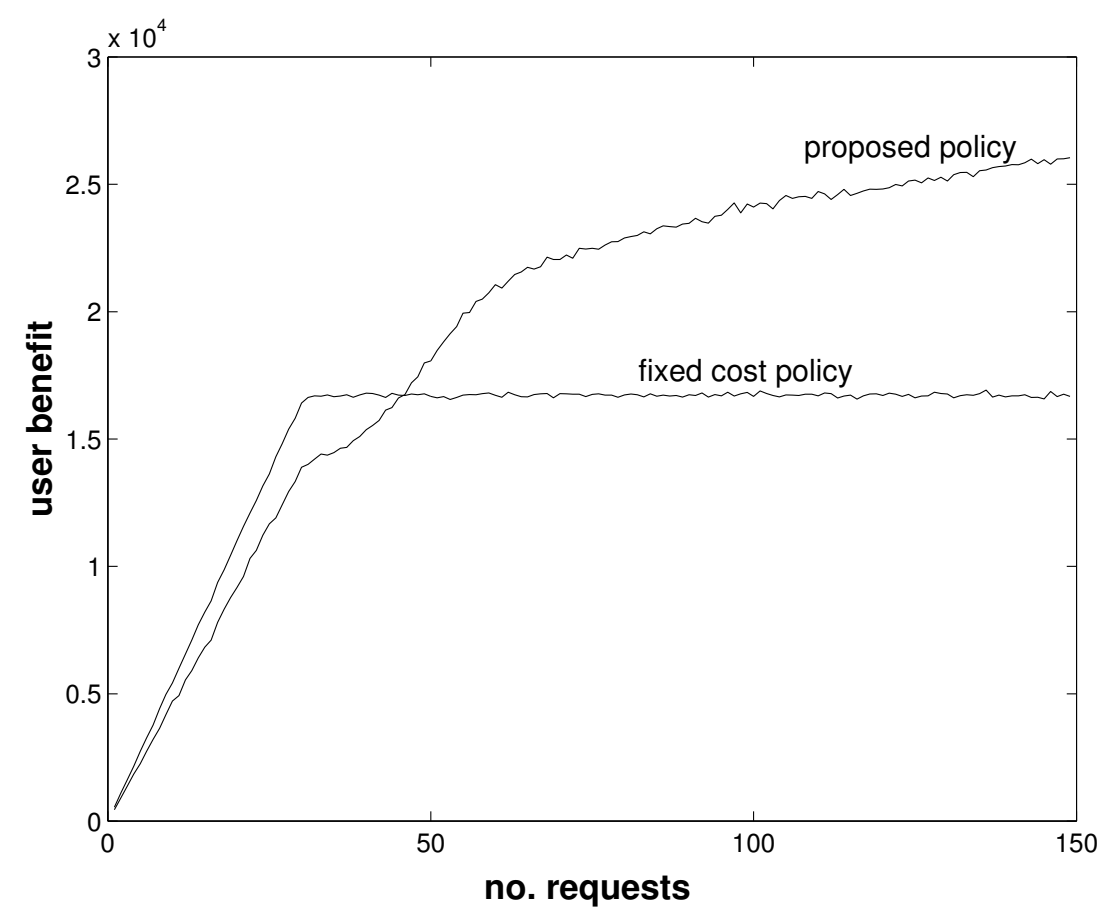

Figure 5: User Benefit: Static case

proposed policy uses application scalability for gains in network connectivity, revenue, and user benefit.

In the above experiments, we assumed that the user always provides the network with the entire range of scalability $\left(b_{l}, b_{h}\right)$. Provision of this range makes the application liable to scaling and consequently to performance degradation. Users will not provide this range unless it optimizes their benefit. In the lightly loaded region, all connections are supported at the $b_{h}$, irrespective of $b_{l}$. Users therefore maximize their benefit by providing the entire range, because this minimizes their cost. In the heavily loaded region, providing the entire range maximizes the probability of connectivity. In the moderately loaded region, user benefit may not be maximized by specifying the whole range. This is indicated by a notch in the user benefit curve in the moderately loaded region (30-50 requests). If users ignore the blocking factor, and assume that bandwidth allocation is always the lower end of the specified range, they optimize by specifying $\left(b_{\min }, b_{h}\right)$ where $b_{\min }$ is calculated as in Equ. 1. Fig. 6 illustrates user benefit with this optimization. As expected, user benefit is lower when the entire range is specified in the lightly and heavily loaded regions. We see that the benefit is greater in the moderately loaded region when the user tries to optimize.

The user may take the blocking factor into account by reducing the optimal value of 


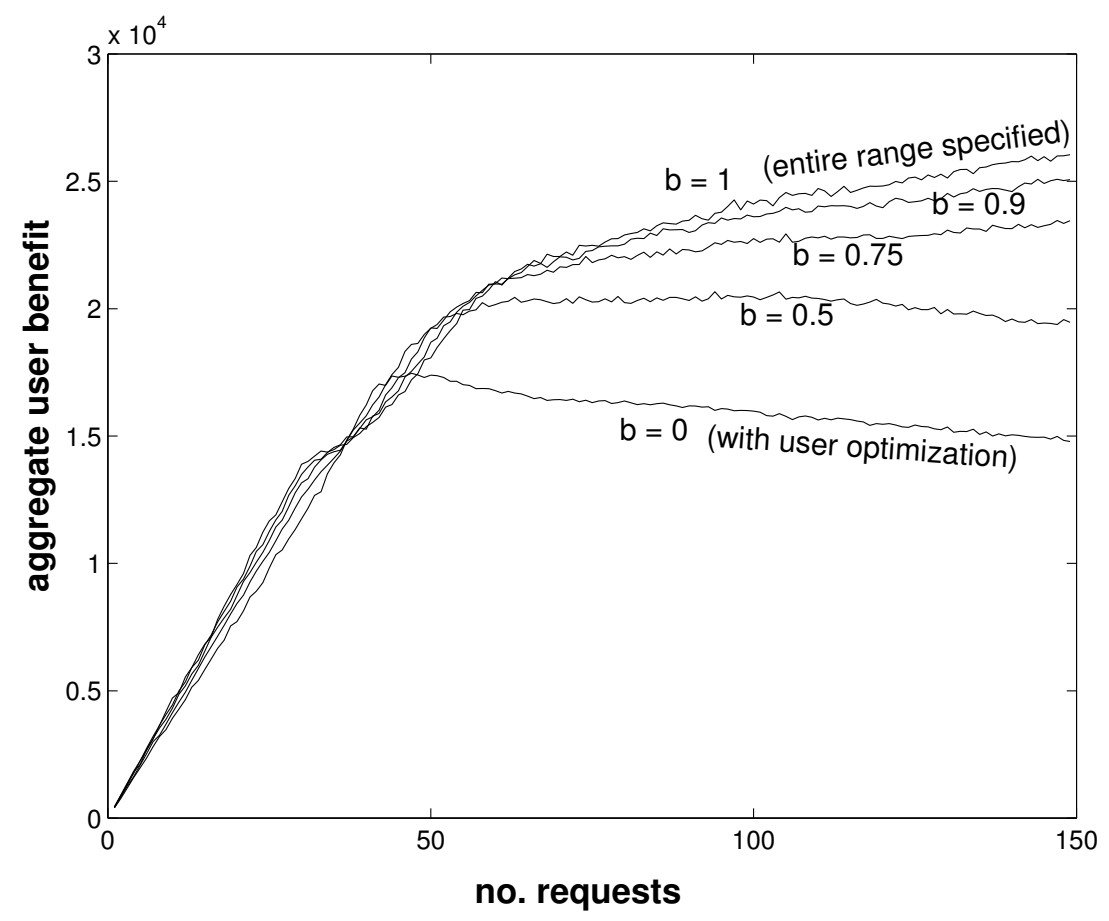

Figure 6: User Benefit with User Optimization: Static case

$b_{\min }$ in Equ. 1 as long as it is above $b_{l}$. We define the back-off factor $b$ such that

$$
b_{\text {min, new }}=b_{\text {min }}-b \times\left(b_{\text {min }}-b_{l}\right)
$$

Fig. 6 shows the benefit curves for different values of $b$. The envelope of these curves is the optimal user benefit curve and would be obtained if the user could calculate the optimal range knowing the load in the network taking the blocking factor into account. Note that the notch is not completely straightened out in the envelope curve. This is because the optimal value of $b_{\min }$ lies outside the $\left(b_{l}, b_{h}\right)$ range for some requests. Thus, in a static scenario, a region exists where an optimizing user does not specify the entire range to the network. We note that this region is small and the gains may not be significant enough to offset the complexity and overheads introduced by the optimizing algorithm. The existence of this region in the dynamic scenario is investigated next.

Though the study of the static scenario provided us with insight into user behavior and system performance, the dynamic scenario models an actual network more closely. In the dynamic case, the network has to make a decision on connection admittance when each request is received. Once a connection is admitted, it has to be allocated at least the minimum specified bandwidth for its duration. We expect a degradation in performance as 
compared to the static case since the network cannot rank connections before deciding which ones to admit. We assume that in the fixed cost case, the available bandwidth $b_{a}$ is known. If $b_{a}$ is less than $b_{h}$, the connection demands the larger of $b_{a}$ and $b_{l}$. In the proposed policy, the entire range $\left(b_{l}, b_{h}\right)$ is specified. Fig. 7 shows that the fixed cost policy starts blocking

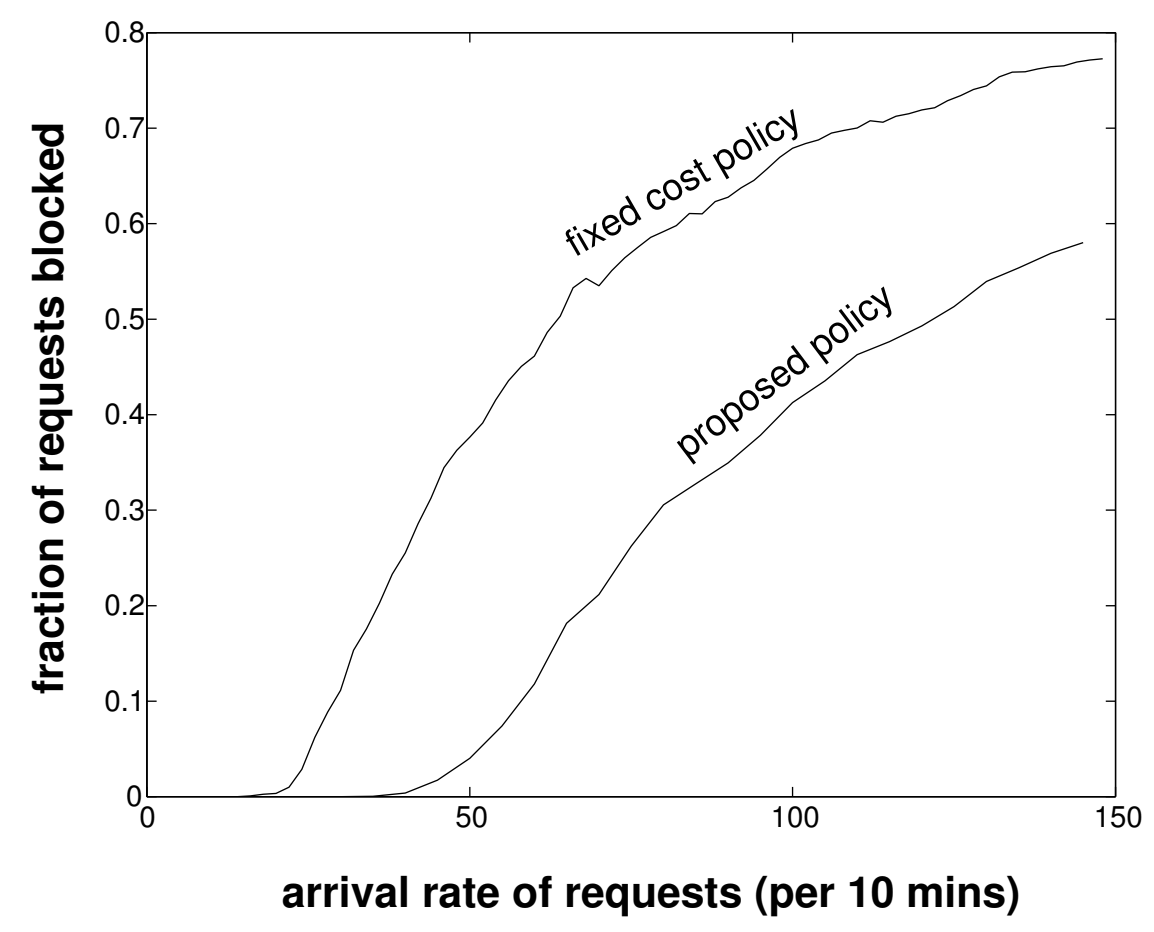

Figure 7: Percentage of Blocked Requests (Dynamic Case)

at a much lower number of requests than the proposed policy. In both cases, requests are admitted as they are received until there is no more bandwidth left. Beyond this, the fixed cost policy blocks requests, while the proposed policy scales down the admitted connections to free up bandwidth for the new request. The network revenue increases in the blocking region for the proposed policy because connections are still admitted if sufficient bandwidth is released by scaling existing connections. In the fixed cost case, revenue flattens out as the number of admitted connections increases only slightly. This is illustrated in Fig. 8.

A similar trend is observed in the user benefit curves shown in Fig. 9. In the fixed cost case, few additional connections are accepted after blocking starts. The utility of admitted connections decreases while the cost stays the same, so the utility flattens out. In the proposed policy, the loss of utility when a connection is scaled is offset by the decrease in cost. Furthermore, new connections are still accepted in the blocking region. 


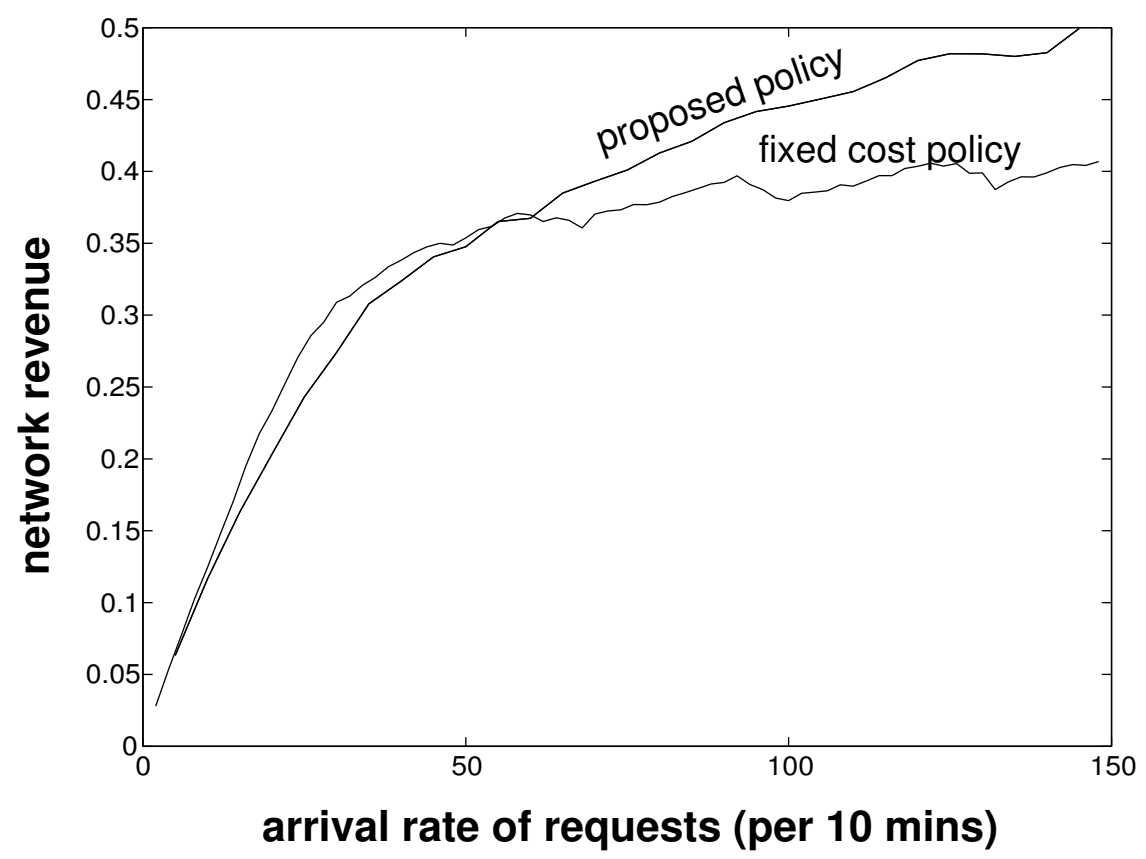

Figure 8: Network Revenue: Dynamic Case

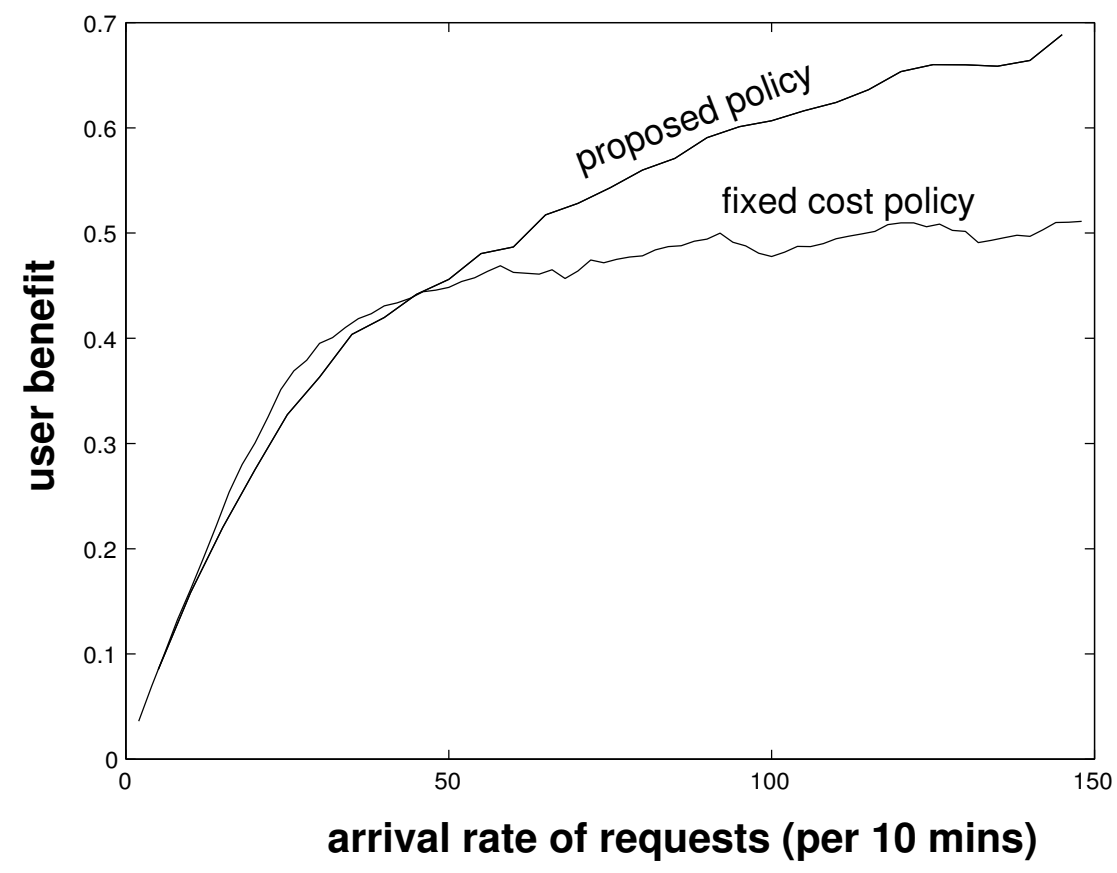

Figure 9: User Benefit: Dynamic Case 
Finally, we examine user preferences for the proposed policy in the dynamic case. Fig. 10 shows user benefit curves for three user preferences. Curve B corresponds to a policy

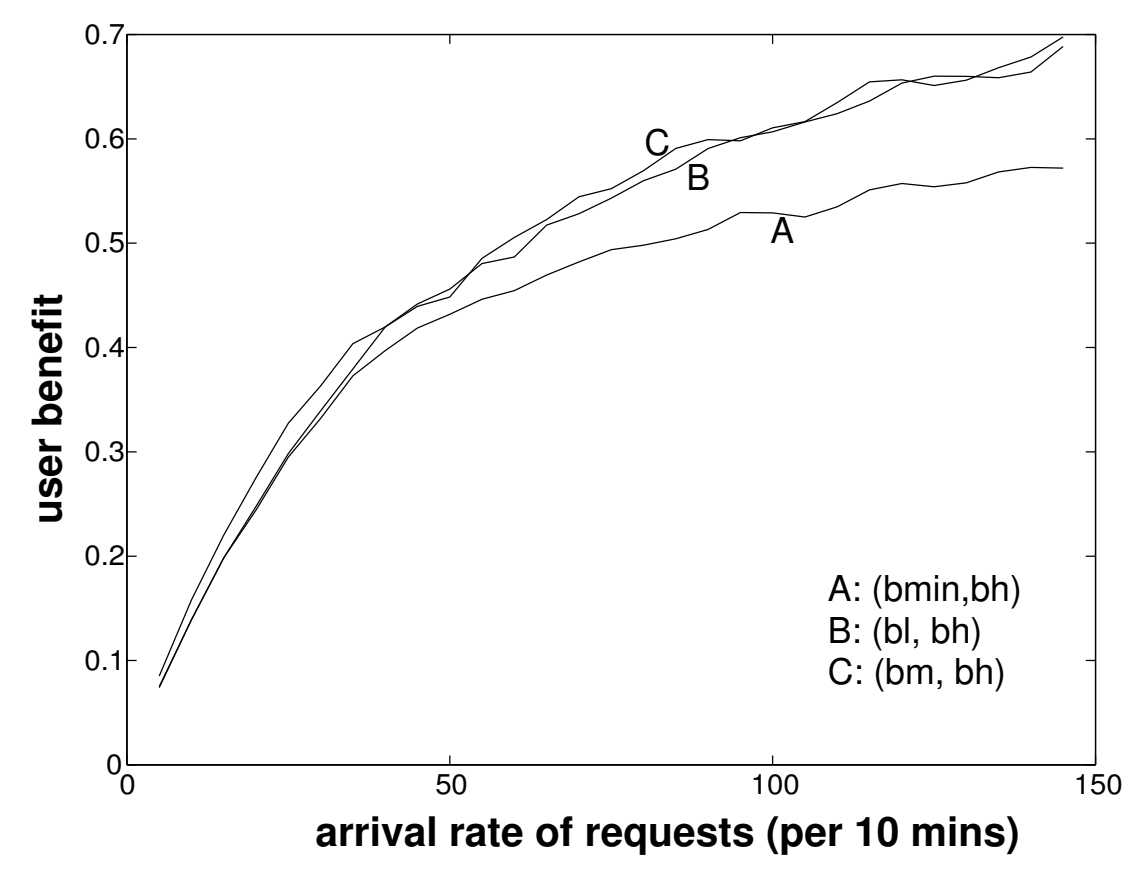

Figure 10: User Benefit With User Optimization: Dynamic Case

where the user specifies the entire range $\left(b_{l}, b_{h}\right)$. Curve A corresponds to the specification of $\left(b_{\min }, b_{h}\right)$, where $b_{\min }$ is calculated using $(1)$. The user ignores the probability of blockage in the specification of minimum bandwidth, and this curve is clearly suboptimal. The third policy (Curve C) assumes that the user has information about the available bandwidth $b_{a}$ and specifies $\left(b_{m}, b_{h}\right)$ where $b_{m}=\max \left(\left(\min \left(b_{\min }, b_{a}\right)\right), b_{l}\right)$. That is, the optimal bandwidth is specified only if it is less than the available bandwidth. Otherwise, the available bandwidth is specified, lower bounded by $b_{l}$. Curve $\mathrm{C}$ is optimistic in that the user gets information on maximum bandwidth available for admission. We observe that Curve B is close to Curve $\mathrm{C}$, suggesting that users should provide near full scalability. This implies that even if the user tailors the specification based on knowledge about available bandwidth, the benefit does not improve significantly in the blocking region. ${ }^{10}$ Thus, the user achieves close to optimal benefit by specifying the entire range of scalability to the network.

\footnotetext{
${ }^{10}$ Note that $B$ is optimal in the lightly loaded region.
} 
Complexity The advantages of a dynamic resource reservation scheme are accompanied by increased overheads. In a static scheme, reservations are fixed once they have been allocated at set-up time. In a dynamic scheme, resources have to be reallocated every time a connection is scaled up or down. Fig. 11 shows the average number of times connections are scaled during their lifetime (10 mins). In the lightly loaded region, all connections are

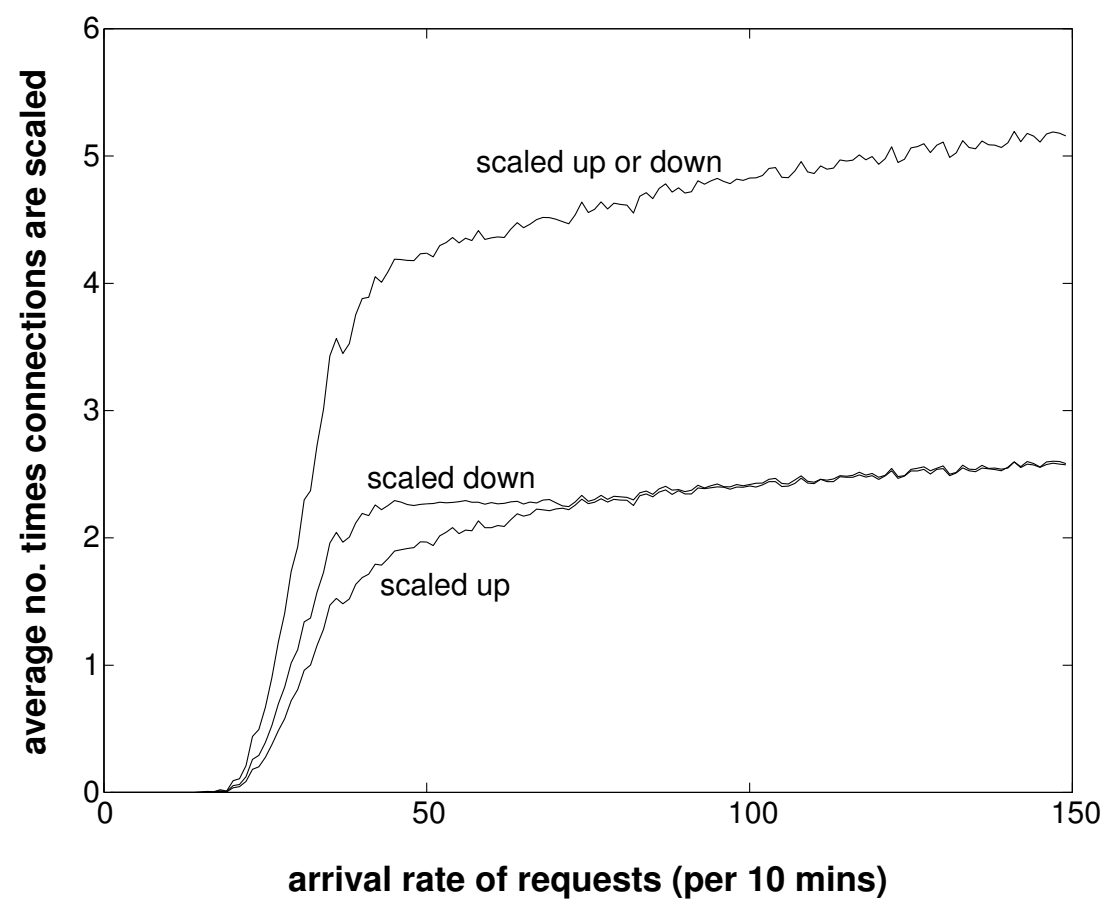

Figure 11: Average Frequency of Connection Scaling

admitted at $b_{h}$ and never scaled down. As the load increases, connections are scaled down to admit new requests. Connections are also scaled up every time a connection is released. In the heavily loaded regions, connections are admitted at their minimum bandwidth, and are scaled up when a connection is released. However, they are scaled down immediately when new requests arrive. Thus the frequency of scaling up and down is almost equal in this region. In the moderately loaded region, frequency of down-scaling is greater than that of up-scaling since connections may be admitted at the maximum bandwidth and then scaled down to admit new connections. We observe that the average number of times connections are scaled during their lifetime is about 5 . This means that connections are scaled on average every 120 seconds. This compares favorably to the renegotiation period of about 20 seconds suggested and found feasible by Zhang et al. [12]. The worst case overhead is obtained by studying the maximum times a connection is scaled, illustrated in Fig. 12. We see that the 


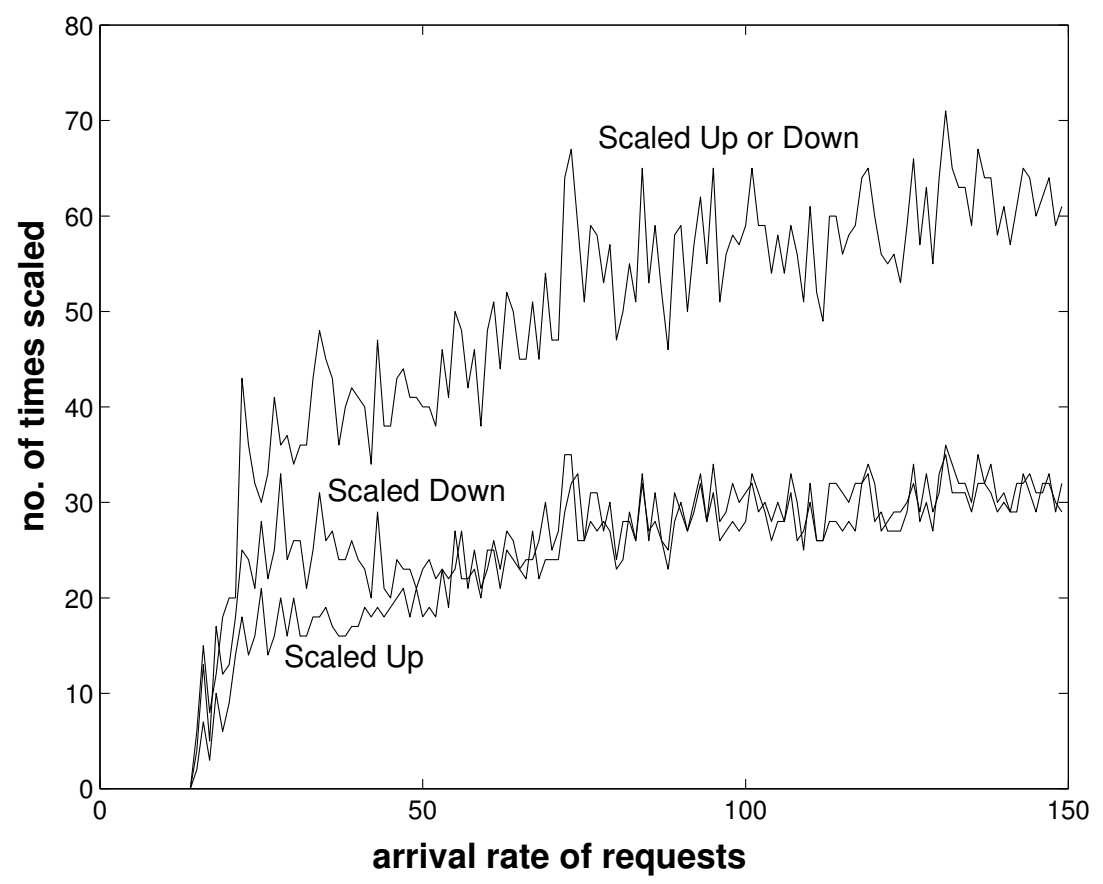

Figure 12: Average Frequency of Connection Scaling

maximum number of times connections are scaled is about 70, meaning that in the worst case, a connection was scaled once every 8.6 seconds. This is not an unreasonable worst case overhead.

Other Pricing Schemes In our study, we have compared the fixed cost policy with our proposed policy. We now briefly discuss two other pricing policies.

In a fixed price rate policy, the cost is proportional to the bandwidth used.

$$
C(x)= \begin{cases}P \times x, & \text { if } b w_{l}<x \\ 0, & \text { otherwise }\end{cases}
$$

where $P$ is the price per unit bandwidth. In this case, the network obtains the same revenue per unit bandwidth irrespective of the connection that receives the allocation. Therefore, there is no incentive for the network to prefer one connection over another. For optimum user benefit, we need

$$
\begin{gathered}
U^{\prime}\left(x_{o p t}\right)=P \\
x_{o p t}=b w_{h}-P .
\end{gathered}
$$

As the price increases, users demand fewer resources to maximize their benefit. When $x_{o p t}$ falls below $b w_{l}$, the user asks for $b w_{l}$. We observe that the user has no incentive to specify 
scalability to the network. This case is therefore similar to the fixed cost case. The blocking rate will be lower than in the fixed cost case because users demand less than the maximum bandwidth. However, performance gains due to scaling are not obtained.

Another possible policy uses a variable rate. Here, the price of the resource depends on the amount of resource demanded, i.e., $P=P(x)$. Typically, $P$ is a convex function of $x$; the price of the resource increases with the amount of resources purchased. For example,

$$
P(x)=\frac{x^{2}}{2}
$$

Such a policy discourages users from demanding very high bandwidths unless they are willing to face a non-linear increase in cost. The admission control policy maximizes revenue by allowing connections with higher bandwidth requirements, obtaining more revenue per unit bandwidth. Thus, users may not improve their chances of connectivity by specifying lower requirements. For optimal benefit,

$$
\begin{gathered}
U^{\prime}\left(x_{o p t}\right)=P^{\prime}(x) \\
b_{h}-x_{o p t}=P \times x_{o p t} \\
x_{o p t}=\frac{b_{h}}{1+P}
\end{gathered}
$$

Again, users have no incentive to specify scalability to the network.

The preliminary analysis above indicates that none of the existing pricing schemes considered here provide incentives for the user to specify scalability to the network. Additional analysis and simulations for these policies will be considered in future work.

\section{Conclusions}

Most video applications are scalable. The network can apply this scalability to improve connectivity and revenue by means of a dynamic resource reservation protocol. However, users suffer from performance degradation when the connection is scaled down. Thus, users will not specify scalability to the network unless there is an incentive to do so. In this paper we propose a pricing policy which provides users with monetary incentives to specify scalability. We also proposed a corresponding dynamic admission control scheme which the network uses to maximize its revenue. Our simulation results demonstrate that the proposed pricing policy encourages users to specify application scalability to the network during connection establishment by increasing their benefit. We also show that this policy, coupled with the admission control scheme, improves user utility, network revenue, and network connectivity over a fixed cost scheme which does not consider application scalability. 


\section{References}

[1] Cocchi, R., S. Shenker, D. Estrin, L. Zhang, "Pricing in Computer Networks: Motivation, Formulation, and Example," IEEE/ACM Trans. on Networking, Vol. 1, No. 6, 1993, pp. 614-627.

[2] Delgrossi, L., C. Halstrick, D. Hehmann, R. Herrtwich, O. Krone, J. Sandvoss, and C. Vogt, "Media Scaling for Audio Visual Communication with the Heidelberg Transport System," Proc. ACM Multimedia '93, Anaheim, CA, August, 1993, pp. 99-104.

[3] Ferrari, D. and D.C. Verma, "A Scheme for Real-Time Channel Establishment in WideArea Networks," IEEE Journal on Selected Areas in Communications, Vol. 8, No. 3, pp. 368-379, April 1990.

[4] Krishnamurthy, A., "A Dynamic Resource Reservation and Pricing Mechanism for Scalable Video Delivery," Ph.D. Dissertation, Deptartment of Electrical, Computer and Systems Engineering, Boston University, 1995.

[5] Krishnamurthy, A. and T.D.C. Little, "Connection-Oriented Service Renegotiation for Scalable Video Delivery," Proc. IEEE Intl. Conf. on Multimedia Computing and Systems, Boston, MA, May 1994, pp. 502-507.

[6] Krishnamurthy, A., T.D.C. Little and D. Castañon, "A Pricing Policy for Scalable VOD Applications," Proc. IEEE Second Intl. Workshop on Community Networking Integrated Multimedia Services to the Home, Princeton, NJ, June 1995, pp. 139-146.

[7] MacKie-Mason, J.K., and H. Varian, "Pricing the Internet, in Public Access to the Internet, B. Kahin, J. Keller (eds.), MIT Press, , CAmbridge, MA, 1995.

[8] Parris, C. and D. Ferrari, "A Resource Based Pricing Policy for Real-Time Channels in a Packet-Switching Network," Technical Report TR-92-018, International Computer Science Institute, Berkeley, California, 1992.

[9] Parris, C., S. Keshav, and D. Ferrari, "A Framework for the Study of Pricing in Integrated Networks," Technical Report TR-92-016, International Computer Science Institute, Berkeley, California, 1992.

[10] Sairamesh, J., D.F. Ferguson, and Y. Yemini, "An Approach to Pricing, Optimal Allocation and Quality of Service Provisioning in High Speed Networks," Proc. IEEE Infocom '95, Boston, MA, pp. 1111-1119. 
[11] Shenker, S., "Service Models and Pricing Policies for an Integrated Services Network," Proc. Public Access to the Internet, Harvard University, Cambridge, MA, 1993.

[12] Zhang, H. and E. Knightly, "RED-VBR: A New Approach to Support Delay-Sensitive VBR Video in Packet-Switched Networks," in Lecture Notes in Computer Science, Vol. 1018, T.D.C. Little and R. Gusella (eds.), Springer, 1995, pp. 258-272. 